\title{
L'HOMME L'Homme
}

Revue française d'anthropologie

165 | janvier-mars 2003

Image et anthropologie

\section{Giulia Sissa, L'Âme est un corps de femme}

Paris, Éditions Odile Jacob, 2000, 224 p.

\section{Patrick Kaplanian}

\section{CpenEdition}

Journals

Édition électronique

URL : http://journals.openedition.org/lhomme/16082

DOI : 10.4000//homme.16082

ISSN : 1953-8103

Éditeur

Éditions de l'EHESS

\section{Édition imprimée}

Date de publication : 1 janvier 2003

Pagination : 347-348

ISBN : 2-7132-1779-2

ISSN : 0439-4216

Référence électronique

Patrick Kaplanian, "Giulia Sissa, L'Âme est un corps de femme », L'Homme [En ligne], 165 | janvier-mars 2003, mis en ligne le 27 mars 2008, consulté le 23 septembre 2020. URL : http://

journals.openedition.org//homme/16082 ; DOI : https://doi.org/10.4000//homme.16082

Ce document a été généré automatiquement le 23 septembre 2020.

(c) École des hautes études en sciences sociales 


\title{
Giulia Sissa, L'Âme est un corps de femme
}

Paris, Éditions Odile Jacob, 2000, 224 p.

\author{
Patrick Kaplanian
}

1 L'ÂME est un corps. C'est ce que démontre la troisième partie. Ainsi, lorsque Socrate se défend lors de son procès, il amène son adversaire à se contredire, provoquant chez lui une honte dont l'effet est purificateur, à l'image d'une katharsis du corps. De même, chez les sceptiques, la connaissance du monde extérieur se heurte à la barrière du corps nécessaire à son appréhension. Tout ce que je peux dire c'est que la neige me parait blanche, le miel sucré. Un homme n'aura pas la même impression que tel ou tel animal, ni qu'un autre homme, ni que lui-même en d'autres circonstances. « Dire qu'on ne devrait jamais affirmer que le miel est sucré mais seulement qu'il semble sucré, cela signifie s'astreindre à ne jamais parler du monde, mais exclusivement de soi-même, [...] c'est-à-dire de ce que le corps ressent » (p. 154). Aussi l'affectivité prime-t-elle sur la connaissance, car il n'y a pas de connaissance absolue, mais seulement des impressions (p. 159). Ici aussi on retrouve l'image du corps : les arguments sceptiques ne prétendent pas remplacer des propositions fausses par des propositions vraies, «ils agissent comme des médicaments purgatifs qui provoquent l'évacuation des substances pathogènes, et s'écoulent eux-mêmes » (pp. 155-156) ; ils fonctionnent donc comme des potions cathartiques qui laissent les entrailles vides. "Nous reconnaissons ici ", conclut l'auteur, «l'analogie entre nettoyage de l'âme et purification du corps par vidange et élimination d'un contenu encombrant » (p. 156).

2 L'âme est un corps de femme. C'est l'objet de la seconde partie. On connaît la maieutique, cet art de l'accouchement que pratique Socrate. Mais, et c'est là l'originalité, l'apport le plus important de ce livre, l'âme-corps de femme n'est pas grosse de la vérité, mais seulement d'idées fausses. Finalement - il faut suivre ici la démonstration très serrée de l'auteur -, la maieutique du célèbre dialogue qui la décrit, le Théétète, n'aide à se libérer que d'idées fausses, d'œufs de vent. Il s'agit de faire le vide, de faire place nette, bref, une fois de plus, de katharsis. Toutefois Platon ne se 
contente pas du scepticisme: la connaissance est ailleurs, dans la théorie de l'anamnèse.

3 Cette image féminine de l'âme enceinte d'idées fausses s'appuie sur la conception qu'on se faisait de la femme à l'époque: "Faute d'accéder réellement à l'éducation, les femmes incarnent dans l'imaginaire une accessibilité, une perméabilité presque sans résistance [...], cohérente avec leur vocation sexuelle à accueillir, à prendre en soi » (pp. 19-20). Le champ du sexuel est ainsi élargi : est de l'ordre du féminin ce qui est capable de recevoir n'importe quoi. Cela explique peut-être que la maieutique est une métaphore de l'accouchement, mais de l'accouchement seul. Sont face à face la femme et la sage-femme; le concepteur est absent. L'approche d'une belle âme, c'est-à-dire d'un philosophe, provoque le déclic purificatoire. Le philosophe n'est donc pas à la place du concepteur.

4 Mais ce n'est pas toujours la règle : la Pythie que décrit Plutarque doit aussi être pure, vierge dans tous les sens du mot, absente de toute souillure et d'idées fausses. Ici, la conception joue son rôle puisque, une fois purifiée, elle peut recevoir la parole du dieu (Apollon). Elle conçoit par les œuvres du dieu et accouche de sa parole.

5 C'est peut-être la première partie qui intéressera le plus le lecteur ethnologue. Elle traite de la conception grecque de la différence des sexes. Le féminin grec se pense par contraste et par défaut, jamais en soi, presque jamais dans une comparaison qui lui serait favorable. La femme est passive et inférieure à l'homme. Pour Platon, tout ce que les hommes font, les femmes le font moins bien. Certes, Socrate reconnait dans la République que les femmes sont les plus douées pour le tissage et la cuisine, mais ce sont choses négligeables. Ainsi Platon nie-t-il la division sexuelle du travail. Ou du moins souhaite-t-il qu'elle disparaisse dans sa République. Mais comment faire si les femmes sont par nature inférieures? Pour Aristote, cette infériorité est systématique: anatomique, physiologique, éthique. Lacunes, moindre qualité, inadéquation, mutilation, incomplétude.

6 Les deux philosophes se heurtent à des problèmes de taxinomie. Genos (genre) et eidos (espèce) n'ont pas grand-chose à voir avec les classifications modernes. Chez Platon (et probablement déjà chez Hésiode, vers $700 \mathrm{av}$. J.-C., qui parle de genos des femmes), les deux concepts sont mal distingués. Aristote est perplexe qui voit bien qu'une jument et un étalon appartiennent tous deux au genre cheval, mais qui ne sait que dire des flagrantes différences anatomiques. Il s'en tire en faisant de la femelle « un ratage systématique par rapport à un modèle » (p. 45). Le corps féminin dans son ensemble paraît marqué par une série de traits qui en manifestent la nature défectueuse, faible, incomplète (p. 43). La différence sexuelle est selon le plus ou le moins (p. 45). Ainsi la jument, en tant qu'étalon raté, appartient bien à la même espèce que l'étalon.

7 L'infériorité chez Platon est aussi morale. Dans le Timée, les hommes, à l'origine, étaient heureux. Mais certains, lâches, faibles, malhonnêtes, se réincarnèrent en femmes (p. 36). L'infériorité morale de la femme de Platon reflète ainsi l'infériorité physique de la femme d'Aristote. L'un des aspects de cette infériorité de la femme est donc sa passivité, passivité qui en fait un corps-réceptacle à l'image d'une âme prête à recevoir toutes formes d'idées fausses.

8 Giulia Sissa ajoute une comparaison avec les poètes de l'époque archaïque (pp. 36-37) qui lui permet de déduire que cette infériorité physique, morale voire professionnelle, remonterait aux origines de la Grèce. Je n'en suis pas sûr. La femme d'Hésiode (vers 700 av. J.-C.) est bien méchante, avide, égoïste. Mais Hésiode définit ces traits négatifs 
" positivement ». Pandore, la première femme, est fabriquée de toutes pièces, elle n'est pas un homme dégradé, diminué, dégénéré. Et le modèle utilisé pour la fabriquer est une déesse.

9 Ce livre montre l'intérêt que peut avoir l'éclairage ethnologique sur le discours philosophique. Considérés à l'aune de la conception grecque de l'identité sexuelle, les textes de Platon, d'Aristote ou des sceptiques prennent une coloration différente de celle que leur donne l'histoire de la philosophie.

\section{AUTEUR}

\section{PATRICK KAPLANIAN}

Paris. 